\section{Prevalencia y factores asociados al consumo de cigarrillo tradicional, en adolescentes escolarizados}

\author{
JAVIER MARTÍNEZ-TORRES ${ }^{1, \mathrm{a}}$, MARTHA PEÑUELA EPALZA ${ }^{2, \mathrm{~b}}$
}

\section{Prevalence of smoking among Colombian adolescents}

\begin{abstract}
Background: Cigarette smoking is considered the most important preventable public health problem in developed countries, especially among adolescents. Aim: To determine the prevalence of cigarette smoking and associated factors in high school adolescents, from a Colombian city. Material and Methods: The self-administered global tobacco youth survey (GTYS) was answered by 831 teenagers aged $14 \pm 2$ years (54\% females). For data analysis, proportions were calculated; for associations, binary and multivariable logistic regression was applied. Results: Fourteen percent of respondents declared that they had consumed at least one cigarette during the last 30 days. The life-time prevalence of tobacco use was $27.1 \%$. Being older than thirteen years old, father's academic level and having a smoker mother were factors associated with smoking. Conclusions: The prevalence of smoking in these adolescents was high. Age over 13 years and a smoking mother were associated with the cigarette smoking.

(Rev Med Chile 2017; 145: 309-318)

Key words: Adolescent; Cross-Sectional Studies; Prevalence; Smoking; Tobacco Use.
\end{abstract}

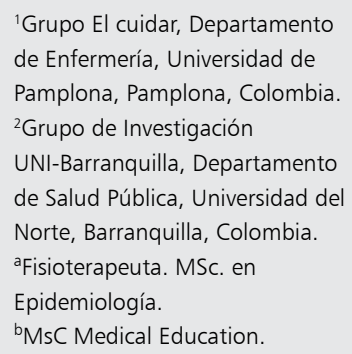

Financiación: No contó con financiación externa, al ser parte de la tesis de maestría del autor principal.

Recibido el 11 de agosto de 2016, aceptado el 7 marzo de 2017.

Correspondencia a: Javier Martínez Torres epid_javier@hotmail.com epidjavier@gmail.com
E 1 consumo de cigarrillos es considerado hoy el problema más grande de salud pública prevenible en países desarrollados. Su uso entre adolescentes constituye uno de los principales problemas de los sistemas de salud pública en el orden global ${ }^{1}$. El consumo de cigarrillos tiene consecuencias no solamente en la salud, sino en la economía. Entre los primeros están la reducción en la esperanza de vida, el desarrollo de enfermedades neurológicas, cardiovasculares y pulmonares y la alteración de los sistemas de defensa bioquímicos que generan cáncer de pulmón $^{2}$. A su vez, la morbilidad asociada incrementa los costos médicos para el sistema ${ }^{3}$, así como los gastos de bolsillo.

El expendio de cigarrillos está prohibido para menores de edad; sin embargo, ellos no tienen impedimentos para adquirirlos. En esa etapa de la vida, los jóvenes están sometidos a presión por el grupo al que pertenecen, por lo que es probable que empiecen a consumir cigarrillos debido a que sus iguales pueden ser persuasivos con ese tema. Los jóvenes comienzan a experimentar con el tabaco a los 10 años de edad y se convierten en fumadores regulares aproximadamente a los $15^{4,5}$. El consumo del cigarrillo, al formar parte de la costumbre social de muchas sociedades lo convierte en un negocio rentable, cuya producción y consumo tienen un impacto sobre los recursos sociales y económicos de todos los países por igual ${ }^{6}$.

El tabaquismo es una drogodependencia con características psicosociales y farmacológicas. La nicotina es el componente del tabaco responsable de la adicción y es diez veces más adictiva que la cocaína y la morfina; puede producir tolerancia y 
dependencia física, que desembocan en un síndrome de privación cuando se intenta la abstinencia ${ }^{7}$. La adicción a la nicotina es la principal razón de que los individuos persistan en el consumo de tabaco, el cual contribuye como causa de muchas enfermedades.

La Encuesta Nacional de Salud de Colombia realizada en el año 2007 mostró que entre los adolescentes de 12 a 17 años la estimación de la categoría fumador actual de tabaco fue de 2,4\%. Para las edades de 18 a 69, años la prevalencia de tabaquismo en hombres se calculó en 19,5\% y en mujeres de $7,4 \%$, con $12,8 \%$ para el total de la población ${ }^{8}$. Se ha demostrado que el consumo de cigarrillos en la edad adulta está fuertemente relacionado con el inicio de ese hábito en la adolescencia, ya que cerca de $82 \%$ de los fumadores actuales tuvieron contacto con ese producto antes de los 18 años, mientras que $16 \%$ lo tuvo cuando tenían entre 18 y 26 años. Es de resaltar que, aproximadamente, 9 de cada 10 consumidores diarios de cigarrillo probaron ese producto antes de los 18 años, y cerca de dos tercios $(65,1 \%)$ de los adultos que fuman todos los días comenzaron a fumar a diario, antes de los 18 años; $y$ apenas alrededor de $4 \%$ empezó a hacerlo después de haber cumplido 26 años 9

Por lo expuesto, surge la necesidad de conocer el comportamiento de consumo de ese producto tabáquico. El objetivo de este trabajo es examinar la prevalencia y factores sociodemográficos asociados al consumo de cigarrillo tradicional en una muestra representativa de estudiantes de secundaria en Pamplona, Norte de Santander, Colombia.

\section{Materiales y Métodos}

\section{Tipo de estudio y población}

Se trata de un estudio descriptivo transversal, que forma parte del estudio "Emtamplona". Para el cálculo de la muestra se tomó como referencia la población matriculada para el 28 de febrero de 2015 (4.118 estudiantes), en los grados sexto a undécimo de los colegios del área urbana de Pamplona. Debido a que no hay estudios que evalúen la prevalencia de dicho evento en Pamplona, se tomó una proporción esperada del 0,50 (50\%), con un margen de error de $3 \%$ y un nivel de confianza del $95 \%$. El muestreo fue estratificado por tipo de colegio y curso.

\section{Procesos de medición y recolección de la información}

La recolección de la información se realizó tomando como base la Emtajoven ${ }^{10}$. Se realizó una encuesta estructurada, autodiligenciada, anónima, a cada uno de los estudiantes se les explicó la importancia de la medición, y siempre estuvo el grupo encuestador pendiente de las inquietudes que presentaban los adolescentes frente a el cuestionario; para evaluar el consumo de cigarrillo actual (consumo durante los últimos 30 días), y el haberlo consumido alguna vez en la vida (lo cual se describe como: «haber probado el cigarrillo»). La valoración del consumo actual de cigarrillo, o de haberlo probado, se hizo por medio de una sola pregunta para cada caso, en la cual el sujeto acusaba con un sí o un no; para el consumo actual se preguntó si había fumado al menos un cigarrillo durante los últimos treinta días; y para evaluar la prevalencia de vida, se preguntó si había consumido al menos un cigarrillo, alguna vez en la vida.

\section{Factores sociodemográficos}

Para el presente análisis se definieron como factores asociados las siguientes variables sociodemográficos: 1) género (masculino y femenino); 2) curso actual; 3 ) edad; 4) tipo de colegio (público y privado); 5) religión (católico, cristiano no católico y otra religión/no creyente); 6) estrato social (estrato I, estrato II y III, y estrato IV y V); 7) grado académico de la madre; 8 ) grado académico del padre (no tiene padre/madre, no sabe, sin educación, hasta primaria, hasta secundaria, educación técnica o tecnológica, pregrado universitario, y postgrado universitario), y 9) consumo de cigarrillo por parte de los padres.

\section{Análisis estadístico}

En primer lugar se realizó un análisis exploratorio para determinar la distribución de frecuencias (medidas de tendencia central y de dispersión para variables cuantitativas), frecuencias relativas (para variables cualitativas). Se realizó la descripción para la prevalencia actual y de vida por variables sociodemográficas. Posterior a eso, para estimar la asociación entre el consumo actual y consumo de vida, con las variables sociodemográficas de los adolescentes (género, curso actual, edad, tipo de colegio, religión, estrato social, grado académico de la madre, grado académico 
del padre), se utilizaron dos modelos de regresión logística binaria.

El primer modelo de regresión logística binaria fue simple (OR no ajustado), el segundo modelo fue multivariable (Modelo $1^{\text {a }} \mathrm{OR}$ ). Se ingresaron al análisis multivariable las variables asociadas al consumo de cigarrillo en el análisis bivariable que mostraron un valor de $\mathrm{p}<0,20$, por criterio de Hosmer Lesmeshow. Los análisis fueron realizados en SPSS versión 20, se consideró como significativo un valor $\mathrm{p}<0,05$, la estimación de parámetros se hizo con un nivel del confianza de 95,0\%.

\section{Consideraciones éticas}

El estudio se desarrolló de acuerdo a las normas establecidas en la Resolución 8.430 de 1993 del Ministerio de Salud de Colombia; de acuerdo con su artículo 11, esta investigación se clasifica como "sin riesgo". La participación fue voluntaria y quienes aceptaron formar parte del estudio firmaron un consentimiento informado. Los datos fueron manejados con estricta confidencialidad y anonimato sin realizarse análisis individuales, el formato de valoración estaba numerado con un código, el cual sólo fue usado en el proceso de tabulación y estaba separado de los registros de consentimiento y asentimiento informado. El estudio fue aprobado por el Comité de Ética de la Universidad del Norte, Acta de evaluación número: 125 del 30 de abril de 2015.

\section{Resultados}

La muestra estuvo constituida por 862 adolescentes, de los cuales se analizaron 831, quienes completaron totalmente el cuestionario $(96,4 \%$ de respuesta). La población de adolescentes escolarizados encuestados en Pamplona provenía principalmente de escuelas públicas $(91,6 \%)$, con edades entre 10 y 19 años, edad promedio de $13,9 \pm 1,9$ (IC95\% 13,8-14,1), en 53,7\% de género femenino. Del total estudiado, $111(13,4 \%)$ afirmaron haber consumido al menos un cigarrillo durante los últimos 30 días. Tener 19 años, cursar $11^{\circ}$ grado, pertenecer al sexo masculino y asistir a un colegio de carácter público, mostraron las mayores prevalencias de consumo con $40,0 \%$, $22,8 \%, 14,5 \%$ y $14,1 \%$, respectivamente; los demás valores por variables sociodemográficas se muestran en la Tabla 1.
El 27,1\% de los estudiantes evaluados acusaron haber consumido cigarrillo al menos una vez en la vida. Tener 19 años, cursar $11^{\circ}$ grado, pertenecer al sexo masculino y asistir a un colegio de carácter público, mostraron las mayores prevalencias $(80,0 \%, 54,4 \%, 31,7 \%$ y $28,5 \%)$, respectivamente; los demás valores por variables sociodemográficas se muestran en la Tabla 2.

Luego de ajustar por variables sociodemográficas, el consumo de cigarrillo en el último mes se encontró asociado a una mayor edad, a partir de los trece años (OR: 6,74; IC95\%: 1,30-35,01) $\mathrm{y}$ tuvo un incremento significativo que muestra su mayor valor a los 18 años de edad (OR: 65,62; IC95\%:3,54-462,83). También se encontró asociación con el tener un padre con educación técnica o tecnológica (OR: 4,07; IC95\%: 1,06-15,59); las demás asociaciones con respecto a variables sociodemográficas se muestran en la Tabla 3.

Luego de ajustar el análisis por las variables sociodemográficas, el consumo de cigarrillo en la vida se encontró asociado con la edad a partir de los doce años (OR: 5,41; IC95\%: 1,30-35,01) y se observó un incremento significativo, que alcanzó su mayor valor a los diecinueve años de edad (OR: 118,5; IC95\%: 9,35-150,13). Otra variable asociada con el consumo de cigarrillo en la vida fue el pertenecer a una religión no cristiana o sin religión (OR: 2,30; IC95\%: 1,06-5,02); las demás asociaciones con respecto al resto de variables sociodemográficas se muestran en la Tabla 4.

\section{Discusión}

Este es el primer estudio que examina el consumo de cigarrillo en una muestra significativa de estudiantes en zona de provincia, representativa del Norte de Santander, Colombia. Se observó una prevalencia reportada de uso, de al menos uno durante los últimos 30 días, de 13,4\% en los adolescentes encuestados, cifra considerable en términos de salud pública, que excede el promedio de 10\% reportado para escolares en el Estudio Nacional de Consumo de Sustancias Psicoactivas en Población Escolar, Colombia de $2011^{11}$. Si bien estos resultados son inferiores a los hallados en las dos versiones de la Emtajoven, realizadas en la capital del país ${ }^{12}$ en los años 2001 (32,9\%) y 2007 $(29,8 \%)$; son algo mayores a las reportadas en otros estudios colombianos, desarrollados en ciudades 
Tabla 1. Prevalencia de consumo de cigarrillo en los últimos 30 días por variables sociodemográficas

\begin{tabular}{|c|c|c|c|c|c|}
\hline & Sí ha & umido & No ha & umido & $\mathbf{p}$ \\
\hline & n & $\%$ & n & $\%$ & \\
\hline Total & 111 & 13,4 & 720 & 86,6 & \\
\hline Género & & & & & \\
\hline Masculino & 56 & 14,5 & 329 & 85,5 & \\
\hline Femenino & 55 & 12,3 & 391 & 87,7 & 0,350 \\
\hline Curso & & & & & \\
\hline Sexto & 13 & 8,4 & 142 & 91,6 & \\
\hline Séptimo & 11 & 7,5 & 135 & 92,5 & \\
\hline Octavo & 13 & 8,6 & 139 & 91,4 & \\
\hline Noveno & 19 & 15,1 & 107 & 84,9 & $<0,001$ \\
\hline Décimo & 29 & 21,0 & 109 & 79,0 & \\
\hline Undécimo & 26 & 22,8 & 88 & 77,2 & \\
\hline Edad & & & & & \\
\hline Diez años & 0 & 0,0 & 8 & 100,0 & \\
\hline Once años & 2 & 2,2 & 91 & 97,8 & \\
\hline Doce años & 8 & 6,7 & 112 & 93,3 & \\
\hline Trece años & 10 & 6,7 & 139 & 93,3 & \\
\hline Catorce años & 20 & 16,7 & 100 & 83,3 & \\
\hline Quince años & 20 & 13,0 & 134 & 87,0 & $<0,001$ \\
\hline Dieciséis años & 31 & 25,4 & 91 & 74,6 & \\
\hline Diecisiete años & 11 & 26,8 & 30 & 73,2 & \\
\hline Dieciocho años & 7 & 36,8 & 12 & 63,2 & \\
\hline Diecinueve años & 2 & 40,0 & 3 & 60,0 & \\
\hline Tipo de colegio & & & & & \\
\hline Público & 107 & 14,1 & 654 & 85,9 & \\
\hline Privado & 4 & 5,7 & 66 & 94,3 & 0,049 \\
\hline Religión & & & & & \\
\hline Católico & 95 & 12,9 & 640 & 87,1 & \\
\hline Cristiano no católico & 8 & 12,9 & 54 & 87,1 & 0,205 \\
\hline Otros* & 8 & 23,5 & 26 & 76,5 & \\
\hline Estrato social & & & & & \\
\hline Estrato uno & 51 & 16,8 & 253 & 83,2 & \\
\hline Estrato dos y tres & 52 & 11,7 & 394 & 88,3 & 0,081 \\
\hline Estrato cuatro o superior & 8 & 9,9 & 73 & 90,1 & \\
\hline Madre & & & & & \\
\hline No tiene madre, no sabe, sin educación & 10 & 9,2 & 99 & 90,8 & \\
\hline Hasta primaria & 40 & 19,0 & 170 & 81,0 & \\
\hline Hasta secundaria & 36 & 13,9 & 223 & 86,1 & \\
\hline Educación técnica o tecnológica & 9 & 10,3 & 78 & 89,7 & 0,068 \\
\hline Pregrado universitario & 8 & 9,9 & 73 & 90,1 & \\
\hline Postgrado universitario & 8 & 9,4 & 77 & 90,6 & \\
\hline Padre & & & & & \\
\hline No tiene padre, no sabe, sin educación & 23 & 10,5 & 196 & 89,5 & \\
\hline Hasta primaria & 40 & 19,1 & 169 & 80,9 & \\
\hline Hasta secundaria & 28 & 13,3 & 182 & 86,7 & \\
\hline Educación técnica o tecnológica & 11 & 14,9 & 63 & 85,1 & 0,036 \\
\hline Pregrado universitario & 6 & 10,3 & 52 & 89,7 & \\
\hline Postgrado universitario & 3 & 4,9 & 58 & 95,1 & \\
\hline Padres consumidores & & & & & \\
\hline Ninguno de ellos & 75 & 11,5 & 575 & 88,5 & \\
\hline Los dos (mamá y papá) & 4 & 23,5 & 13 & 76,5 & \\
\hline Sólo papá & 15 & 15,5 & 82 & 84,5 & 0,009 \\
\hline Sólo mamá & 8 & 32,0 & 17 & 68,0 & \\
\hline No sabe & 9 & 21,4 & 33 & 78,6 & \\
\hline
\end{tabular}

*Incluye ateo y personas que aseveraron ser de otras religiones. Valor $\mathrm{p}$ calculado con $\chi^{2}$. 
Tabla 2. Prevalencia de consumo de cigarrillo en la vida por variables sociodemográficas

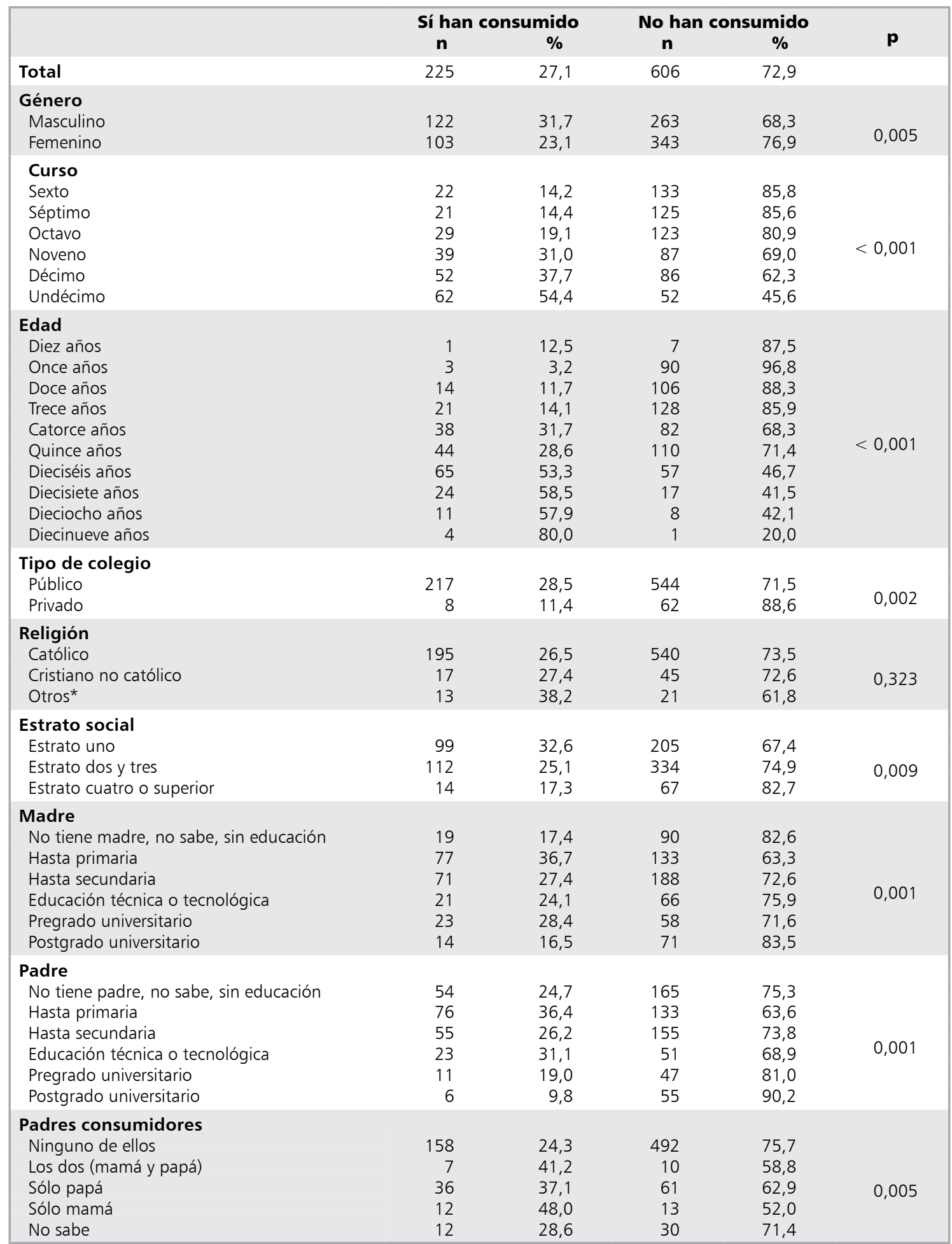

*Incluye ateo y personas que aseveraron ser de otras religiones. Valor $\mathrm{p}$ calculado con $\chi^{2}$. 
Tabla 3. Asociación entre consumo de cigarrillo en los últimos $\mathbf{3 0}$ días y variables sociodemográficas

\begin{tabular}{|c|c|c|c|c|c|c|}
\hline & OR (1) & IC95\% & $\mathbf{p}$ & OR (2) & IC95\% & $\mathbf{p}$ \\
\hline \multicolumn{7}{|l|}{ Género (a) } \\
\hline Masculino & 1,21 & $0,81-1,81$ & 0,874 & 1,11 & $0,72-1,72$ & 0,749 \\
\hline \multicolumn{7}{|l|}{ Curso (b) } \\
\hline Sexto & 1,12 & $0,49-2,60$ & 0,782 & 1,99 & $0,80-4,95$ & 0,567 \\
\hline Octavo & 1,15 & $0,50-2,66$ & 0,746 & 0,72 & $0,26-1,97$ & 0,626 \\
\hline Noveno & 2,18 & $0,99-4,78$ & 0,051 & 0,76 & $0,27-2,14$ & 0,356 \\
\hline Décimo & 3,27 & $1,56-6,84$ & 0,011 & 1,06 & $0,36-3,13$ & 0,898 \\
\hline Undécimo & 3,63 & $1,70-7,72$ & 0,001 & 0,69 & $0,22-2,21$ & 0,723 \\
\hline \multicolumn{7}{|l|}{ Edad (c) } \\
\hline Diez años & N.A. & N.A. & -- & N.A. & N.A. & -- \\
\hline Doce años & 3,25 & $0,67-15,74$ & 0,122 & 4,58 & $0,93-22,55$ & 0,087 \\
\hline Trece años & 3,27 & $0,70-15.33$ & 0,119 & 6,74 & $1,30-35,01$ & 0,023 \\
\hline Catorce años & 9,10 & $2,06-40,16$ & 0,001 & 17,79 & $3,57-88,61$ & 0,001 \\
\hline Quince años & 6,79 & $1,55-29,86$ & 0,001 & 12,44 & $2,38-65,0$ & 0,001 \\
\hline Dieciséis años & 15,50 & $3,59-66,89$ & 0,001 & 33,10 & $6,15,178,13$ & 0,001 \\
\hline Diecisiete años & 16,68 & $3,49-79,82$ & 0,001 & 33,78 & $5,74-198,80$ & 0,001 \\
\hline Dieciocho años & 26,54 & $4,92-143,31$ & 0,001 & 65,62 & $9,19-468,75$ & 0,001 \\
\hline Diecinueve años & 30,33 & 3,11-295,72 & 0,001 & 40,46 & $3,54-462,83$ & 0,001 \\
\hline \multicolumn{7}{|l|}{ Tipo de colegio (d) } \\
\hline Público & 2,70 & $0,96-7,58$ & 0,053 & 1,63 & $0,40-6,56$ & 0,565 \\
\hline \multicolumn{7}{|l|}{ Religión (e) } \\
\hline Católico & 1,00 & $0,46-2,17$ & 0,996 & 1,06 & $0,44-2,52$ & 0,876 \\
\hline Otros* & 2,08 & $0,70-6,17$ & 0,186 & 3,33 & $0,96-11,61$ & 0,099 \\
\hline \multicolumn{7}{|l|}{ Estrato social (f) } \\
\hline Estrato uno & 1,84 & $0,80-34,06$ & 0,126 & 0,70 & $0,24-2,06$ & 0,134 \\
\hline Estrato dos y tres & 1,20 & $0,55-2,65$ & 0,642 & 0,64 & $0,22-1,80$ & 0,545 \\
\hline \multicolumn{7}{|l|}{ Madre (g) } \\
\hline No tiene madre, no sabe, sin educación & 0,97 & $0,37-2,59$ & 0,945 & 0,74 & $0,25-2,22$ & 0,768 \\
\hline Hasta primaria & 2,27 & $1,01-5,08$ & 0,042 & 1,08 & $0,45-2,62$ & 0,653 \\
\hline Hasta secundaria & 1,55 & $0,69-3,49$ & 0,284 & 0,86 & $0,36-2,02$ & 0,457 \\
\hline Educación técnica o tecnológica & 1,11 & $0,41-3,03$ & 0,837 & 0,55 & $0,201,51$ & 0,876 \\
\hline Pregrado universitario & 1,06 & $0,38-2,96$ & 0,738 & 0,61 & $0,20-1,86$ & 0,789 \\
\hline \multicolumn{7}{|l|}{ Padre (g) } \\
\hline No tiene padre, no sabe, sin educación & 2,27 & $0,66-7,5$ & 0,183 & 2,22 & $0,65-7,63$ & 0,234 \\
\hline Hasta primaria & 4,58 & $1,36-15,39$ & 0,007 & 3,44 & $0,99-11,95$ & 0,065 \\
\hline Hasta secundaria & 2,97 & $0,87-10,17$ & 0,069 & 2,92 & $0,85-9,99$ & 0,079 \\
\hline Educación técnica o tecnológica & 3,38 & $0,89-12,74$ & 0,059 & 4,07 & $1,06-15,59$ & 0,017 \\
\hline Pregrado universitario & 2,23 & $0,53-9,40$ & 0,405 & 2,58 & $0,58-11,58$ & 0,389 \\
\hline \multicolumn{7}{|l|}{ Padres consumidores (h) } \\
\hline Los dos (mamá y papá) & 2,36 & $0,75-7,44$ & 0,131 & 2,11 & $0,61-7,27$ & 0,203 \\
\hline Sólo papá & 1,40 & $0,77-2,56$ & 0,267 & 1,35 & $0,68-2,68$ & 0,347 \\
\hline Sólo mamá & 3,61 & $1,50-8,66$ & 0,002 & 5,15 & $1,81-14,71$ & 0,001 \\
\hline No sabe & 2,09 & $0,96-4,55$ & 0,057 & 1,74 & $0,73-4,13$ & 0,174 \\
\hline
\end{tabular}

Grupo de referencia: (a) Femenino; (b) Séptimo; (c) Once años; (d) privado; (e) cristiano no católico; (f) Estrato cuatro o superior; (g) Posgrado universitario; (h) ninguno de los dos. OR (1): Modelo bivariable; OR (2) Modelo multivariable. Los valores estadísticamente significativos se muestran en negrilla. N.A. No aplica, debido a que el factor tiene una prevalencia de $0,0 \%$. *Incluye ateo y personas que aseveraron ser de otras religiones. 
Tabla 4. Asociación entre consumo de cigarrillo en la vida y variables sociodemográficas

\begin{tabular}{|c|c|c|c|c|c|c|}
\hline & OR (1) & IC95\% & $p$ & OR (2) & IC95\% & $p$ \\
\hline \multicolumn{7}{|l|}{ Género (a) } \\
\hline Masculino & 1,55 & $1,14-2,10$ & 0,005 & 1,68 & $1,17-242$ & 0,001 \\
\hline \multicolumn{7}{|l|}{ Curso (b) } \\
\hline Séptimo & 1,02 & $0,53-1,94$ & 0,887 & 0,57 & $0,27-1,22$ & 0,345 \\
\hline Octavo & 1,43 & $0,78-2,62$ & 0,252 & 0,53 & $0,24-1,18$ & 0,389 \\
\hline Noveno & 2,71 & $1,50-4,98$ & 0,001 & 0,47 & $0,21-1,05$ & 0,423 \\
\hline Décimo & 3,66 & $2,07-6,46$ & 0,001 & 0,59 & $0,25-1,41$ & 0,323 \\
\hline Undécimo & 7,21 & $4,02-12,92$ & 0,001 & 0,69 & $0,27-1,77$ & 0,298 \\
\hline \multicolumn{7}{|l|}{ Edad (c) } \\
\hline Diez años & 4,29 & $0,39-47,02$ & 0,196 & 5,97 & $0,46-77,61$ & 0,578 \\
\hline Doce años & 3,96 & $1,10-14,26$ & 0,024 & 5,41 & $1,44-20,33$ & 0,001 \\
\hline Trece años & 4,92 & $1,42-17,04$ & 0,006 & 9,31 & $2,4635,39$ & 0,001 \\
\hline Catorce años & 13,90 & $4,12-46,87$ & 0,001 & 23,38 & $6,20-88,19$ & 0,001 \\
\hline Quince años & 12,00 & $3,60-40,03$ & 0,001 & 20,30 & $5,21-79,21$ & 0,001 \\
\hline Dieciséis años & 34,21 & $10,24-114,32$ & 0,001 & 55,29 & $13,58-225,0$ & 0,001 \\
\hline Diecisiete años & 42,35 & $11,43-156,98$ & 0,001 & 61,08 & $12,99-287,1$ & 0,001 \\
\hline Dieciocho años & 41,25 & $9,48-179,44$ & 0,001 & 56,28 & $10,20-310,5$ & 0,001 \\
\hline Diecinueve años & 120,00 & $10,05-1432,9$ & 0,001 & 118,50 & $9,35-1501,3$ & 0,001 \\
\hline \multicolumn{7}{|l|}{ Tipo de colegio (d) } \\
\hline Público & 3,09 & $1,45-6,57$ & 0,002 & 1,85 & $0,69-4,98$ & 0,238 \\
\hline \multicolumn{7}{|l|}{ Religión (e) } \\
\hline Cristiano no católico & 1,05 & $0,58-1,87$ & 0,879 & 0,98 & $0,48-22,01$ & 0,602 \\
\hline Otros* & 1,71 & $0,84-3,50$ & 0,133 & 2,30 & $1,06-5,02$ & 0,041 \\
\hline \multicolumn{7}{|l|}{ Estrato social (f) } \\
\hline Estrato uno & 2,31 & $1,24-4,32$ & 0,036 & 0,94 & $0,41-2,18$ & 0,738 \\
\hline Estrato dos y tres & 1,61 & $0,87-2,97$ & 0,325 & 0,90 & $0,41-2,00$ & 0,792 \\
\hline \multicolumn{7}{|l|}{ Madre (g) } \\
\hline No tiene madre, no sabe, sin educación & 1,07 & $0,50-2,29$ & 0,859 & 0,71 & $0,29-1,77$ & 0,692 \\
\hline Hasta Primaria & 2,94 & $1,55-5,57$ & 0,001 & 1,41 & $0,63-3,16$ & 0,239 \\
\hline Hasta secundaria & 1,92 & $1,01-3,62$ & 0,042 & 1,09 & $0,50-2,37$ & 0,801 \\
\hline Educación técnica o tecnológica & 1,61 & $0,76-3,44$ & 0,015 & 0,80 & $0,33-1,94$ & 0,670 \\
\hline Pregrado universitario & 2,01 & $0,95-4,26$ & 0,065 & 1,55 & $0,66-3,64$ & 0,652 \\
\hline \multicolumn{7}{|l|}{ Padre (g) } \\
\hline No tiene padre, no sabe, sin educación & 3,00 & $1,22-7,37$ & 0,013 & 2,69 & $1,02-7,09$ & 0,043 \\
\hline Hasta primaria & 5,24 & $2,15-12,76$ & 0,001 & 3,20 & $1,19-8,63$ & 0,032 \\
\hline Hasta secundaria & 3,25 & $1,32-7,99$ & 0,007 & 2,57 & $0,96-6,89$ & 0,078 \\
\hline Educación técnica o tecnológica & 4,13 & $1,56-10,99$ & 0,003 & 4,04 & $1,39-11,72$ & 0,001 \\
\hline Pregrado universitario & 2,15 & $0,74-6,36$ & 0,154 & 1,80 & $0,58-5,65$ & 0,398 \\
\hline \multicolumn{7}{|l|}{ Padres consumidores (h) } \\
\hline Los dos (mamá y papá) & 2,18 & $0,81-5,83$ & 0,116 & 2,60 & $0,83-8,21$ & 0,129 \\
\hline Sólo papá & 1,84 & $1,17-2,88$ & 0,007 & 2,05 & $1,19-3,54$ & 0,021 \\
\hline Sólo mamá & 2,87 & $1,28-6,44$ & 0,008 & 5,16 & $1,82-14,67$ & 0,001 \\
\hline No sabe & 1,25 & $0,62-2,49$ & 0,533 & 1,03 & $0,47-2,26$ & 0,879 \\
\hline
\end{tabular}

Grupo de referencia: (a) Femenino; (b) Sexto; (c) Once años; (d) privado; (e) cristiano católico; (f) Estrato cuatro o superior; (g) Posgrado universitario; (h) Ninguno de los dos. OR (1): Modelo bivariable; OR (2) Modelo multivariable. Los valores estadísticamente significativos se muestran en negrilla. *Incluye ateo y personas que aseveraron ser de otras religiones. 
como Cartagena ${ }^{13}$, Ebéjico, Antioquia ${ }^{14}$ y Bucaramanga ${ }^{15}$ con prevalencias de $6,9 \%, 10,8 \%$ y $11,1 \%$, respectivamente. Los resultados de la aplicación de la Emtajoven en otros países de Sudamérica muestran prevalencias con diferentes valores; en Venezuela fue de $6,8 \%{ }^{16} \mathrm{y}$ en Brasil de $14,3 \%{ }^{17}$.

Otros estudios internacionales, que no se basan en la Emtajoven, muestran diversas prevalencia de consumo de tabaco en adolescentes: en Chile en 2004 se encontró una prevalencia de $33,04 \%{ }^{18}$ y en 2007 fue de $39,9 \%{ }^{19}$, en México es de $23,9 \%{ }^{20}$, en Brasil de $12,2 \%{ }^{21}$, en España, de $28,9 \%{ }^{22}$; mientras que en varios países de Europa oriental oscila entre $5,6 \%$ y $33,1 \%{ }^{23}$; en Lituania, aproximadamente el $30 \%{ }^{24}$, en Corea del Sur, de 5,0\% ${ }^{25}$; en Mongolia, de $9,2 \%{ }^{26}$; en Florida, Estados Unidos de Norteamérica, de $7,1 \%{ }^{27}$. La prevalencia más alta de consumo de todo el mundo se ha encontrado en Ucrania con $60,6 \%{ }^{28}$.

La prevalencia de consumo en el último mes por género mostró que los hombres tienen 2,2 puntos porcentuales por encima de las mujeres, quienes tienen $12,3 \%$. Estas diferencias no son estadísticamente significativas y concuerdan con los hallazgos de Ortiz et al en el $2009^{29}$, y de Birri et al en adolescentes argentinos ${ }^{30}$. Por el contrario, Pitarque et al muestran que la prevalencia en varones es mayor ${ }^{31}$; dos estudios en población universitaria muestran tendencias similares ${ }^{32,33}$, e internacionalmente se conserva dicha tenden$\mathrm{cia}^{21,24-26,34-36}$. En general, hay suficiente evidencia que respalda el planteamiento de que el sexo masculino tiene mayor prevalencia; desde 1994 López et $\mathrm{al}^{37}$ plantearon un modelo descriptivo sobre la epidemia del consumo en el que el sexo masculino mostraría mayores prevalencias en todas las etapas. Sin embargo, dos estudios en Suecia, en $2007^{38}$ y $2015^{35}$, muestran que la prevalencia es mayor en adolescentes mujeres.

Con relación al tipo de colegio, los del sector público mostraron una prevalencia de $14,1 \%$, que es 2,5 veces superior a la hallada en colegios privados. Estos resultados concuerdan con los hallados por Puente et $\mathrm{al}^{22}$ en España. En Cartagena se conserva dicha tendencia, cabe resaltar que la prevalencia en los colegios públicos es, aproximadamente, 50,0\% mayor $^{39}$.

La prevalencia del consumo de cigarrillos en el último mes aumenta con la edad, pues pasan de 2,2\% en los jóvenes de 11 años y llegan a 40\% en los de 19 años. Similar tendencia se ha encon- trado en estudios realizados en Argentina, donde a medida que los adolescentes aumentan su edad también crece el porcentaje de consumo ${ }^{31}$. Un estudio desarrollado en Brasil ${ }^{21}$ muestra que entre los 10 y los 13 años la prevalencia es de $1,2 \%$, y asciende a $10,9 \%$ entre los 14 y los 16 años; la prevalencia alcanza su tope entre los 17 y los 19 años, con $25,8 \%$. Resultados similares se han obtenido en estudios internacionales ${ }^{17,20,24-26,34-36}$.

El consumo de cigarrillos entre adolescentes que aseveraron no ser adeptos al cristianismo mostraron la mayor prevalencia, con 23,5\%; aunque no se encontraron estudios que analizaran dicho consumo por creencia religiosa en adolescentes, estos resultados son acordes con los hallados por Martínez et al. ${ }^{33}$, que determinaron que entre la población universitaria las personas ateas tenían mayor proporción de consumo.

Varios estudios han relacionado el consumo de cigarrillos por adolescentes con la pertenencia a clases sociales bajas. Uno de ellos fue realizado en España ${ }^{22}$ y otro en la costa colombiana, el cual muestra que es $30 \%$ más alta en estratos sociales bajos, comparados con estratos altos ${ }^{39}$. Este estudio mostró esa tendencia, pues el estrato uno presentó la mayor prevalencia (16,8\%).

El nivel académico de los padres mostró que aquellos adolescentes cuyos padres tenían estudios de postgrado presentaban las menores prevalencias, tanto en madres como en padres, con $9,4 \%$ y $4,9 \%$, respectivamente. Estos resultados concuerdan con los hallados por Malcom et al. ${ }^{21}$ y por Rachiotis et al. ${ }^{36}$, quienes descubrieron que a mayor cantidad de años académicos en los padres, la prevalencia es menor en los adolescentes.

El círculo familiar es un importante factor relacionado con el consumo de cigarrillos. Los resultados muestran que aquellos adolescentes cuyos padres no fuman (ninguno de los dos) tienen la menor prevalencia y que por el contrario, cuando ambos padres fuman, hay una mayor prevalencia $^{22}$. En la población estudiada, si bien se observó una menor prevalencia de consumo entre los adolescentes cuyos padres no fumaban, la mayor prevalencia se encontró entre quienes acusaron que la madre era quien fumaba; resultados que concuerdan con dos estudios suecos desarrollados en el $2007^{38}$ y $2014^{35}$ donde mostraron que las prevalencias son mayores cuando se tiene un padre o madre fumadora; y similar tendencia se encontró en Grecia $^{36}$, Lituania ${ }^{24}$, Zambia $^{40}$. 
En conclusión, la prevalencia de consumo de tabaco es alta en la población estudiada, y factores determinantes como la edad superior a trece años y consumo por parte la madre permanecieron asociados en el análisis ajustado. Estos datos podrían utilizarse para ofrecer programas de intervención educativa acerca de los riesgos del consumo de tabaco.

\section{Referencias}

1. García O, Suárez R, Santonja F, Secades R, Sánchez E. Psychosocial risk factors for adolescent smoking: A school-based study. Int J Clin Health Psychol 2011; 11(1): 23-33.

2. Das S. Harmful health effects of cigarette smoking. Mol Cell Biochem 2003; 253 (1-2): 159-65.

3. Ginsberg G, Geva H. The burden of smoking in Israel-attributable mortality and costs (2014). Isr J Health Policy Res 2014; 3 (1): 28-37.

4. The Global Youth Tobacco Survey Collaborative Group. Tobacco use among youth: a cross country comparison. Tobacco Control 2002; 11 (3): 252-70.

5. DiFranza J, Wellman R, Sargent J, Weitzman M, Hipple $\mathrm{B}$, Winickoff J. Tobacco promotion and the initiation of tobacco use: assessing the evidence for causality. Pediatrics 2006; 117(6): 1237-48.

6. The World Bank. Curbing the epidemic: Governments and the economics of tobacco control. Tobacco Control 1999; 8: 196-201.

7. Soto-Mas F, Villalbí JR, Balcázar H, Valderrama-Alberola J. La iniciación al tabaquismo: aportaciones de la epidemiología, el laboratorio y las ciencias del comportamiento. An Esp Pediatr 2002; 57 (4): 327-33.

8. Rodríguez J, Ruiz F, Peñaloza E, Eslava J, Gómez L, Sánchez H, et al. Encuesta Nacional de Salud 2007. Bogotá; 2009.

9. U.S. Department of Health and Human Services. Substance Abuse and Mental Health Services Administration. National Survey on Drug Use and Health: Summary of National Findings, Results from the 2010. U.S. Department of Health and Human Services; 2011.

10. World Health Association. Tobacco Free Initiative (TFI). [Online]; 2010 (citado el 28 de septiembre de 2014). Available from: http://www.who.int/tobacco/ surveillance/gyts/en/\#.

11. Observatorio de Drogas de Colombia. Estudio Nacional de Consumo de Sustancias Psicoactivas en Población Escolar 2011. Bogotá: Ministerio de Justicia y del Derecho; 2011.
12. Pardo C, Piñeros M, Jones N, Warren C. Results of global youth tobacco surveys in public schools in Bogotá, Colombia. J Sch Health 2010; 80 (3): 141-5.

13. Cogollo Z, Gómez E, Campo A. Consumo de cigarrillo entre estudiantes de Cartagena, Colombia: factores familiares asociados. Rev Fac Nac Salud Pública 2009; 27 (3): 259-63.

14. Betancour A, Zambrano R. Prevalencia del consumo de tabaco en una muestra de adolescentes escolarizados del municipio de Ebéjico, departamento de Antioquia, Colombia. Liber 2014; 20 (1): 131-9.

15. Martínez J, Amaya W, Campillo H, Campo A, LA D. Factores asociados con el consumo diario de cigarrillo en adolescentes estudiantes de básica secundaria de Bucaramanga, Colombia. Biomedica 2005; 25 (4): 518 26.

16. Granero R. Primer Informe Encuesta Mundial sobre Tabaquismo en Jóvenes EMTAJOVEN Venezuela. Barquisimeto: Unidad de Investigación Clínica y Epidemiológica ASCARDIO, Venezuela; 2000.

17. Sánchez Z, Opaleye E, Martins S, Ahluwalia J, Noto A. Adolescent gender differences in the determinants of tobacco smoking: a cross sectional survey among high school students in São Paulo. BMC Public Health 2010; 10 (12): 748-57.

18. Valdivia CG, Simonetti BF, Cumsille EP, Ramírez CV, Hidalgo CC, Palma OB, et al. Consumo de tabaco en población menor de 18 años: estudio de prevalencia en escolares de Chile. Rev Med Chile 2004; 13 (2): 171-82.

19. Gaete J, Ortúzar C, Zitko P, Montgomery P, Araya R. Influence of school-related factors on smoking among Chilean adolescents: a cross-sectional multilevel study. BMC Pediatr 2016; 16: 79.

20. Nuño B, Álvarez J, Madrigal E, Rasmussen B. Prevalencia y factores asociados al consumo de tabaco en adolescentes de una preparatoria de Guadalajara, Jalisco, México. Salud Mental 2005; 28 (5): 64-70.

21. Malcon M, Menezes A, Chatkin M. Prevalência e fatores de risco para tabagismo em adolescentes. Rev Saude Publica 2003; 37 (1): 1-7.

22. Puente D, Zabaleta-Del-Olmo E, Pueyo M, Saltó E, Marsal J, Bolíbar B. Prevalencia y factores asociados al consumo de tabaco en alumnos de enseñanza secundaria de Cataluña. Aten Primaria 2013; 45 (6): 315-23.

23. Stojiljkovic D, Haralanova M, Nikogosian H, Petrea I, Chauvin J, Warren C, et al. Prevalence of tobacco use among students aged 13-15 years in the South-Eastern Europe health network. Am J Health Behav 2008; 32 (4): 438-45.

24. Jamison B, Muula A, Siziya S, Graham S, Rudatsikira E. Cigarette smoking among school-going adolescents in 
Lithuania: Results from the 2005 Global Youth Tobacco Survey. BMC Res Note 2010; 7 (3): 130.

25. Rudatsikira E, Muula A, Siziya S. Prevalence, correlates of and perceptions toward cigarette smoking among adolescents in South Korea. Indian J Pediatr 209; 76 (5): 505-10.

26. Rudatsikira E, Dondog J, Siziya S, Muula A. Prevalence and determinants of adolescent cigarette smoking in Mongolia. Singapore Med J 2008; 49 (1): 57-62.

27. Barnett T, Soule E, Forrest J, Porter L, Tomar S. Adolescent Electronic Cigarette Use: Associations With Conventional Cigarette and Hookah Smoking. Am J Prev Med 2015; 49 (2): 199-206.

28. Hazemba A, Siziya S, Muula A, Rudatsikira E. Gender specific factors associated with having stopped smoking among in-school adolescents in Ukraine: results from the Global Youth Tobacco Survey 2005. BMC Res Notes 2010; 3: 76.

29. Ortiz-Gómez Y, Rodríguez J. Prevalencia y características del consumo de tabaco en adolescentes del colegio universitario Santiago de Cali. Rev Fac Med Unal 2009; 59 (2): 89-99.

30. Birri M, Cabral M, Agnese A. The prevalence of adolescent smoking in the city of Cordoba. Ars Pharm 2007; 48 (2): 121-34.

31. Pitarque R, Bolzán A, Gatella M, Moranga F, Bugasen $\mathrm{S}$, Echaide L. Tabaquismo en adolescentes escolarizados de la ciudad de Olavarría, Buenos Aires. Prevalencia y factores asociados. Arch Argent Pediatr 2007; 105 (2): 115-21.

32. Castaño-Castrillón J, Páez Cala M, Pinzón Montes J, Rojo Bustamante E, Sánchez-Castrillón G, Torres Ríos J, et al. Estudio descriptivo sobre tabaquismo en la comunidad estudiantil de la universidad de manizales. 2007. Rev Fac Med 2008; 56 (4): 302-17.

33. Martínez-Torres J, Pérez J, Bucheli L, Manrique LCR, Rojas Z, Vargas M. Prevalencia y factores de riesgo del consumo de cigarrillo para estudiantes universitarios entre 18 a 25 años, durante el primer semestre académico del año 2013. Rev Med Unab 2013; 16 (1): 13-8.

34. Mendoza R, López P. El consumo de tabaco en el alumnado español pre-adolescente y adolescente: diferencias de género. Adicciones 2007; 19 (4): 341-55.

35. Hedman L, Andersson M, Stridsman C, Rönmark E. Evaluation of a tobacco prevention programme among teenagers in Sweden. BMJ Open 2015; 5(5): 1-7.

36. Rachiotis G, Muula A, Rudatsikira E, Siziya SKA, Gourgoulianis K, Hadjichristodoulou C. Factors associated with adolescent cigarette smoking in Greece: results from a cross sectional study (GYTS Study). BMC Public Health 2008; 8 (9): 313-19.

37. López A, Collishaw N, Piha T. A descriptive model of the cigarette epidemic in developed countries. Tobacco Control 1994; 3 (3): 242-7.

38. Hedman L, Bjerg A, Perzanowski M, Sundberg S, Rönmark E. Factors related to tobacco use among teenagers. Respir Med 2007; 10 (3): 496-502.

39. Cogollo-Milanés Z, De la Hoz Restrepo F. Consumo de cigarrillo y riesgo de dependencia de la nicotina de estudiantes de secundaria. Rev Salud Pública 2010; 12 (3): 434-45.

40. Siziya S, Rudatsikira E, Muula A. Cigarette smoking among school-going adolescents in Kafue, Zambia. Malawi Med J 2007; 19 (2): 75-8. 